\title{
Why Condition-Based Regression Analysis (CRA) is Indeed a Valid Test of Self-Enhancement Effects: A Response to Krueger et al. (2017)
}

\author{
Sarah Humberg*, Michael Dufner ${ }^{\dagger}$, Felix D. Schönbrodt ${ }^{\ddagger}$, Katharina Geukes*, Roos \\ Hutteman§, Maarten H. W. van Zalk\|, Jaap J. A. Denissen", Steffen Nestler ${ }^{\dagger}$ and Mitja D. \\ Back $^{*}$
}

\begin{abstract}
How can the consequences of self-enhancement (SE) be tested empirically? Traditional two-step approaches for investigating SE effects have been criticized for providing systematically biased results. Recently, we suggested condition-based regression analysis (CRA) as an approach that enables users to test SE effects while overcoming the shortcomings of previous methods. Krueger et al. (2017) reiterated the problems of previous two-step approaches and criticized the extent to which CRA could solve these problems. However, their critique was based on a misrepresentation of our approach: Whereas a key element of CRA is the requirement that the coefficients of a multiple regression model must meet two conditions, Krueger et al.'s argumentation referred to the test of only a single condition. As a consequence, their reasoning does not allow any conclusions to be drawn about the validity of our approach. In this paper, we clarify these misunderstandings and explain why CRA is a valid approach for investigating the consequences of SE.
\end{abstract}

Keywords: self-view; self-enhancement; discrepancy model; algebraic difference; residual scores

Self-enhancement (SE) is often defined as the degree to which the self-view (e.g., about one's ability) exceeds some kind of criterion (e.g., one's objectively measured ability). The (mal)adaptive consequences of SE are one of the most hotly debated topics in social-personality psychology. Are people better (or worse) adjusted the more they overestimate (or the less they underestimate) their positive attributes (e.g., Bonanno, Field, Kovacevic, \& Kaltman, 2002; Church et al., 2006; Colvin, Block, \& Funder, 1995; Dufner, Gebauer, Sedikides, \& Denissen, 2018; Gramzow, Willard, \& Mendes, 2008; Paulhus, 1998; Robins \& Beer, 2001; Sedikides \& Gregg, 2008; Taylor \& Brown, 1988; Taylor, Lerner, Sherman, Sage, \& McDowell, 2003)?

Previous empirical studies addressing such questions have typically applied an analytical approach involving two steps. A discrepancy score (e.g., an algebraic difference or residual) between individuals' self-view and their value

\footnotetext{
* Westfälische Wilhelms-University Münster, DE

+ University of Leipzig, DE

* Ludwig-Maximilians-Universität München, DE

§ Utrecht University, NL

" University of Osnabrück, DE

" University of Tilburg, NL

Corresponding author: Sarah Humberg (sarah@humberg.name)
}

on some criterion measure was computed in a first step, which was then correlated with an outcome variable in a second step. Researchers have repeatedly emphasized that this two-step approach is not appropriate for testing SE effects because it is systematically biased toward mistaking main effects of the self-view for effects of SE (e.g., Asendorpf \& Ostendorf, 1998; Edwards \& Parry, 1993; Griffin, Murray, \& Gonzalez, 1999; Humberg et al., 2018; Krueger \& Wright, 2011; Ullrich, 2009; Zuckerman \& Knee, 1996). Recently, Humberg et al. (2018) introduced condition-based regression analysis (CRA) as an approach that can be applied to overcome the problems of prior two-step approaches. Unlike previous approaches, CRA enables users to test SE effects without mistaking the main effects of the self-view for SE effects. ${ }^{1}$

In a recent article, Krueger, Heck, and Asendorpf (2017) reviewed discrepancy scores that have typically been used in the first step of the analysis of SE effects (see Humberg et al., 2018; Kurt \& Paulhus, 2008; Kwan, John, Kenny, et al., 2004; Kwan, John, Robins, \& Kuang, 2008; Moore \& Healy, 2008; Paulhus, 2008; Robins \& Paulhus, 2001, for similar overviews). The authors then revisited the critique of the two-step approach and concluded that it is not suitable for testing SE effects. We wholeheartedly agree with the authors' critique up to this point in their article: An approach for testing how the discrepancy between self-views and criterion values (i.e., SE) is related to an 
outcome variable should be able to differentiate effects of the discrepancy from mere main effects of the self-view.

After their recap on the statistical problems of twostep approaches, Krueger et al. (2017) noted that CRA has been suggested ${ }^{2}$ as a way to solve these problems, but then they misrepresented the CRA approach and drew a conclusion that would be very unfortunate for the field: They concluded that CRA should be avoided because it suffers from the very same problems as discrepancy score correlations. Specifically, the authors first correctly introduced CRA by stating that two conditions must be tested to detect an SE effect but they then referred to only one of the conditions when evaluating the utility of CRA. Consequently, the argumentation that the authors provided cannot justify their conclusion about CRA. In this rebuttal, we clarify CRA, explain why the critique in Krueger et al. (2017) is unjustified, and explain why CRA does, in fact, solve the problems of prior two-step approaches that have been applied for investigating SE effects.

Note that Krueger et al.'s statistical considerations about discrepancy scores and about CRA constituted only one message presented in their paper. Entangled with their statistical critique, the authors also voiced concern about whether the typically applied conceptualization of SE as a comparison of the self-view and some criterion measure (e.g., a person's self-view vs. this person's rating of the average other; a person's self-view vs. an objective assessment of the ability under consideration) could indeed capture the concept of SE that researchers have aimed to capture. As an alternative, they suggested an approach that categorizes individuals as making a "selfenhancement error" if they believe they have a higherthan-average standing on the ability (i.e., self-view > rating of the average other) when their true standing is in fact below average (i.e., objective ability < average ability in the sample). Our evaluation of these conceptual ideas is that they are worthy of further elaboration. However, these ideas are unrelated to the statistical question of how researchers whose conceptual definition of SE is best reflected in a discrepancy measure (e.g., algebraic difference or residuals) can validly test the association between SE and an outcome variable. We wrote the present article to show that, although Krueger et al. (2017) had indicated otherwise, CRA is an appropriate tool for achieving this aim. We therefore focus on the relevant methodological contents from Krueger et al.'s paper and refer readers who are interested in the authors' conceptual ideas to the original article.

\section{The CRA Approach for Testing SE Effects}

Imagine that we want to test whether people are happier the more they overestimate their cognitive ability, that is, we want to test for a positive SE effect. ${ }^{3}$ We would assess participants' self-ratings $S$ about their ability, some reality criterion $T$ (e.g., intelligence test scores), and happiness $H$ as the outcome variable. We would need to specify what we mean by the term SE, and we could, for example, decide that our understanding of SE is best reflected by the algebraic difference (S-T) between a person's self-rating and his or her criterion value. ${ }^{4}$ In this case, the question of a positive SE effect translates into the question of whether higher values on the discrepancy (S-T) tend to go along with higher values on $\mathrm{H}$. As was repeatedly emphasized in the literature and also described in Krueger et al. (2017), the correlation of (S-T) with $H$ might indicate an SE effect when it is in fact not present (e.g., see Griffin et al., 1999; Ullrich, 2009; Zuckerman \& Knee, 1996) because, inter alia, $\operatorname{Cor}(S-T, H)$ is biased toward mistaking self-view main effects (i.e., an effect of $S$ ) for effects of the difference (S-T).

CRA was designed to solve this problem. It shows whether individual differences in $\mathrm{H}$ are associated with individual differences in (S-T), or, rather, "only" with individual differences in $S$. CRA is based on the linear regression model ${ }^{5}$ that predicts $H$ from $S$ and $T$ :

$$
\mathrm{H}=\beta_{0}+\beta_{1} \mathrm{~S}+\beta_{2} \mathrm{~T}+\mathrm{e}
$$

The key element of CRA is that it states which conditions on the coefficients $\beta_{1}$ and $\beta_{2}$ must be satisfied before we can conclude that there is a positive SE effect. That is, the CRA approach states that the discrepancy (S-T) is positively related to the outcome $H$ if and only if the following two conditions are satisfied (see p. 9 in Humberg et al., 2018):

$$
\beta_{1}>0 \quad \text { and } \quad \beta_{2}<0
$$

For the intuitive derivation of these conditions, we made use of the fact that the coefficient $\beta_{1}$ reflects the effect of $S$ when $T$ is controlled for, and $\beta_{2}$ is the effect of $T$ when $S$ is controlled for. ${ }^{6}$ The first condition ensures that when comparing two people with equal values of ability $T$, the person who has the higher self-view and thus the higher value on the discrepancy $(S-T)$ will be predicted to be happier. The second condition ensures that for two people with equal self-views $S$, the person who has the lower ability level, who is again the person with a higher SE value (S-T), will be predicted to be happier.

Both conditions in Equation 2 must hold before we know that there is a positive SE effect: When the first condition $\beta_{1}>0$ holds but the second condition is violated because $\beta_{2}>0$, the regression model would predict that for two people with the same self-view value, the person with the higher ability level would be happier (because $\beta_{2}>0$ ), but this is in fact the person with lower SE. This situation would contradict a positive SE effect. Similarly, when $\beta_{2}=0$, two people with the same self-view values are predicted to be equally happy, independent of their ability levels and thus independent of their levels of SE. When the first condition is violated (i.e., $\beta_{1}=0$ or $\beta_{1}<0$ ), the model predicts that two people with the same ability level are equally happy independent of their self-views and thus independent of their levels of SE (if $\beta_{1}=0$ ), or it even predicts that the person with the lower self-view and thus the person with lower SE would be happier (if $\beta_{1}<0$ ). All of these scenarios contradict a positive SE effect, indicating that testing only one of the two conditions is not sufficient to test for this effect (see also OSF Material B at osf.io/e8p5r).

The fact that CRA requires that two conditions must be tested before the user can conclude that there is a positive SE effect might seem counterintuitive at first glance. 
However, this requirement can be seen as the key property of CRA because it is the reason why CRA can be applied to overcome the problems of two-step approaches. Intuitively speaking, the two conditions ensure that both sources of information about individual differences in SE, namely, self-views and ability levels, are taken into account in the analysis. Thereby, contrasting CRA with previous approaches, the approach enables researchers to disentangle SE effects from main effects of the self-view.

Instead of testing the two intuitive conditions in Equation 2, an equivalent set of conditions can be tested. This set is less intuitive but provides statistical benefits (i.e., it avoids alpha error accumulation because only the first condition has to be significant, and the second condition has to hold only numerically; see Humberg et al., 2018; see Figure 1). Equivalent to the conditions in Equation 2, the CRA approach states that the discrepancy $(S-T)$ is positively related to the outcome $H$ if and only if the following two conditions are satisfied (see the theorem on p. 10 in Humberg et al., 2018; see OSF Material F at osf.io/e8p5r for the proof that the set of conditions in Equation 3 is equivalent to the set of conditions in Equation 2):

$$
\begin{gathered}
a b s=\left|\beta_{1}-\beta_{2}\right|-\left|\beta_{1}+\beta_{2}\right|>0 \\
\text { and } \quad \beta_{1}-\beta_{2}>0
\end{gathered}
$$

Again, both conditions in Equation 3 must be considered before drawing a conclusion, because none of the conditions considered alone is sufficient for identifying a positive SE effect. For example, when only the second condition $\beta_{1}-\beta_{2}>0$ is tested and satisfied, we only know that $\beta_{1}$ is larger than $\beta_{2}$. It could be the case (Case A) that $\beta_{1}$ is positive and $\beta_{2}$ is negative (indicating a positive SE effect), (Case B) that both $\beta_{1}$ and $\beta_{2}$ are positive (contradicting a positive SE effect), or (Case C) that both coefficients are negative (also contradicting a positive SE effect). If we considered the condition $\beta_{1}-\beta_{2}>0$ to be sufficient to test for a positive SE effect, we would falsely claim support for such an effect in many cases when it is in fact not present (i.e., in Cases B and C). ${ }^{7}$ CRA prevents us from drawing such a false conclusion by requiring that we additionally test the condition $a b s>0$ because this information is needed to conclude whether Case A (if $a b s>0$; positive SE effect) or rather one of the latter cases (Case $\mathrm{B}$ or $\mathrm{C}$ if $a b s \leq 0$; no SE effect) is present.

To sum up, CRA enables researchers to test whether higher values on the discrepancy $(S-T)$, which are often considered a proxy for SE, are associated with higher values on an outcome variable $H$. Testing for a positive SE effect with CRA involves the estimation of a regression model predicting $H$ from $S$ and $T$ and simultaneously testing two conditions on its coefficients or on auxiliary parameters computed from the coefficients (either both conditions stated in Equation 2 or both conditions in Equation 3).

Besides algebraic difference scores, some other operationalizations of SE have been suggested in the literature. For example, researchers have suggested that SE might instead be reflected by residual scores (John \& Robins, 1994) or by the Kwan index (which includes a way to control for rating tendencies; see Kwan et al., 2004). The decision about which SE measure is appropriate for the specific research question at hand should be based on conceptual considerations. In addition, one should take into account the potential pitfalls of discrepancy measures, which were discussed in the long-standing debate on the use of difference scores for measuring change (e.g., the influences of regression toward the mean; for an overview, see Campbell \& Kenny, 1999). Whereas individual researchers should identify an SE measure that accurately

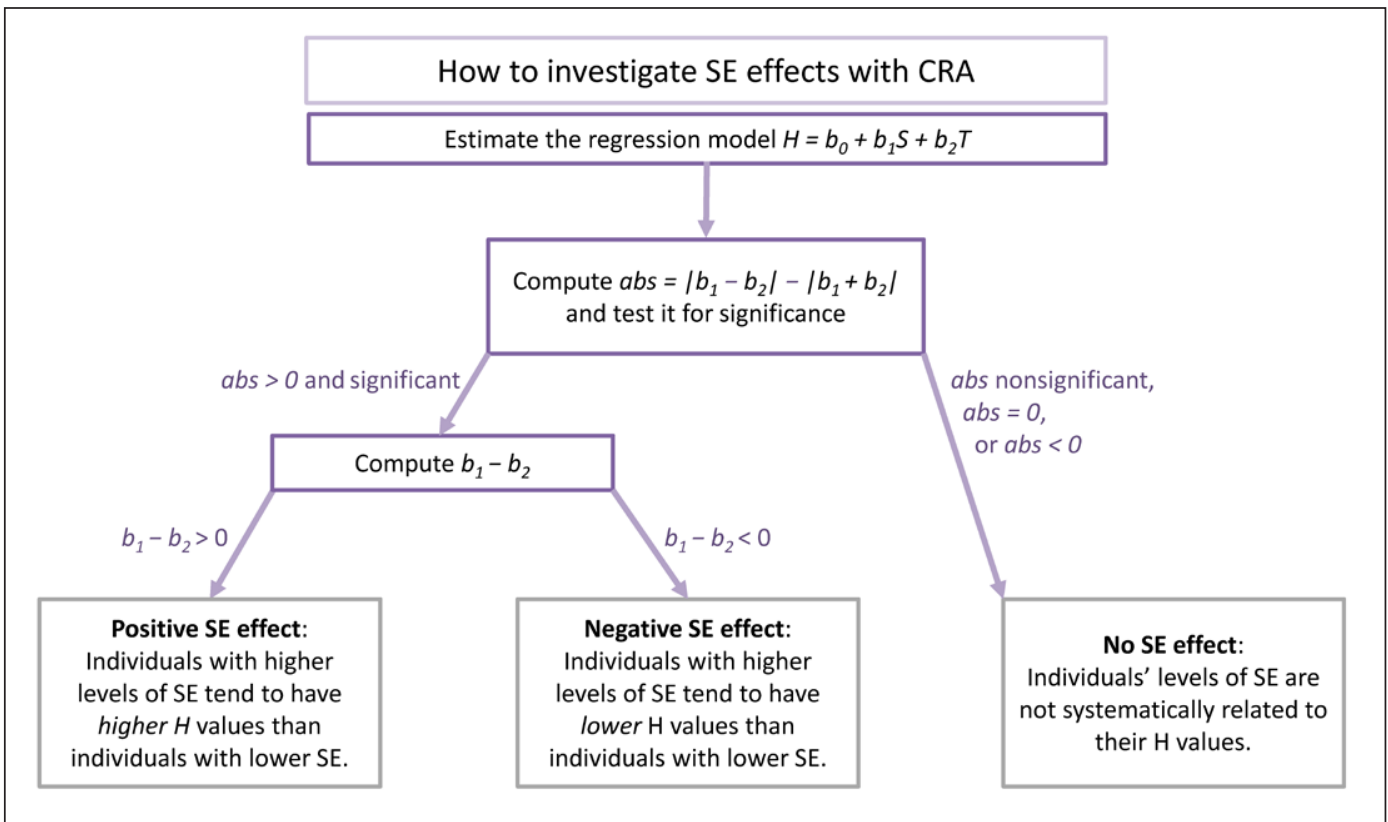

Figure 1: A visual representation of the test of SE effects with CRA. See osf.io/e8p5r for details on the significance test of abs (OSF Material G) and, for example, the R code that demonstrates CRA. The depicted strategy refers to the operationalization of SE as the algebraic difference $S$ - $T$. For different operationalizations, the computation and test of abs must be adapted (see Table 4 in Humberg et al., 2018, see OSF Materials C, D, and E at osf.io/e8p5r). 
reflects the constructs they are interested in, the basic principle of CRA allows them to test SE effects for a variety of possible operationalizations (see Table 4 in Humberg et al., 2018, see OSF Materials C, D, and E at osf.io/e8p5r). This principle is always the same as outlined above: The basic element is a multivariate regression model, and (at least) two conditions must be satisfied for its coefficients before it is possible to conclude that the chosen SE score is positively related to the outcome variable.

An Evaluation of Krueger et al.'s Critique of CRA Krueger et al. (2017) called for an approach that unequivocally identifies SE effects. They reviewed the CRA approach and came to the conclusion that it does not achieve this aim. Their key argument was, basically, that if one considers the condition $\beta_{1}-\beta_{2}>0$ as sufficient to test for a positive SE effect, then one would falsely claim support for such an effect in many cases when it is in fact not present. In the words of the authors, "the difference between the weights $\beta_{1}$ and $\beta_{2}$ is dependent on $\beta_{1}$ much like $S-T$ is dependent on $S$. ... Self-enhancement effects [are] not disentangled from simple self-perception effects" (Krueger et al., 2017, p. 7).

These observations are correct, but they refer exclusively to the test of $\beta_{1}-\beta_{2}$ and thus do not allow any conclusion to be drawn about the validity of CRA. CRA explicitly states that two conditions must be tested before any conclusion can be drawn about a positive SE effect. Moreover, the authors' argument against CRA is fully consistent with one of the central insights provided by the CRA approach, namely, that testing only one condition (e.g., the condition on $\beta_{1}-\beta_{2}$ ) is not sufficient for concluding that there is a positive SE effect. In addition to the condition $\beta_{1}-\beta_{2}>0$, $C R A$ requires $a b s>0$. However, the latter condition for $a b s$, a key element of CRA, is not mentioned at all in Krueger et al.'s review and critique of CRA.

Krueger et al. (2017) mentioned the two conditions required to apply CRA at two points in their article. First, when the authors introduced CRA (p. 6), they initially provided the correct description, which is that, according to the CRA approach, a positive SE effect requires the two conditions $\beta_{1}>0$ and $\beta_{2}<0$ to hold. Afterwards, however, the authors presented the results of simulation studies to demonstrate that testing the difference $\beta_{1}-\beta_{2}$ inherits the same problems as testing the correlation of $(S-T)$ with $H$. Neither their simulations nor their logical reasoning referred to the set of two CRA conditions. ${ }^{8,9}$ This misrepresentation of CRA is also reflected in their statement that "Humberg et al. proposed to let ... the difference between $\beta_{1}$ and a negative $\beta_{2}$ reflect the selfenhancement effect"10 (Krueger et al., 2017, p. 7). This statement is wrong because it is not the difference between the coefficients that is tested in the CRA approach. Second, Krueger et al. (2017) stated that "requiring both regression weights to be significant and of opposite signs, the multiple-regression approach may provide a conservative test for the presence of a self-enhancement effect" (p. 7). The validity of this statement depends on how one interprets the term "conservative": The two conditions ensure that main effects of the self-view are not mistaken for SE effects. Thereby, the CRA approach is "conservative" in the sense that it prevents researchers from drawing systematic false-positive conclusions about their data as they would have done if they had applied any previous two-step approach. However, Krueger et al. (2017) did not integrate this observation into their final evaluation of CRA, but they falsely concluded that CRA was not sufficient for differentiating SE effects from main effects of the self-view. To sum up, although the authors mentioned the two key conditions of CRA at two points in their article, their reasoning exclusively referred to a statistical test that was different from and not equivalent to the CRA approach.

A second concern that Krueger et al. (2017) voiced about CRA refers to terminology: They stated that "the regression weight $\beta_{1}$ is what most traditional researchers consider the self-enhancement effect" (p. 7). Independent of whether this claim is true or not, it is not related to the validity of CRA which tests whether individual differences in an outcome variable $H$ are associated with individual differences in the discrepancy (S-T) (or in other discrepancy scores such as residuals or the Kwan index for the adapted versions of CRA, see Table 4 in Humberg et al., 2018, see OSF Materials C, D, and E at osf.io/e8p5r). Its ability to do so does not depend on the question of how many researchers have thus far been interested in this kind of effect. This being said, we doubt that most researchers have used the term "self-enhancement effect" to refer to self-view main effects while controlling for the criterion, but this is the effect reflected by the coefficient $\beta_{1}$. As can be seen in Krueger et al.'s overview of previous research in this domain, for example, researchers typically operationalized SE as some kind of discrepancy score and stated that higher values on this score should be associated with higher outcome values. In all of these cases, the researchers' methods have implied that they were interested in the effect of a discrepancy score and not in the main effect of self-view. ${ }^{11}$

To sum up, Krueger et al. (2017) questioned whether CRA can provide an appropriate test of SE effects, but their conclusion was based on a misrepresentation of this method as they referred to only one of two necessary conditions stated in the CRA approach. In fact, when applying CRA as originally described in Humberg et al. (2018), this approach actually solves the problems that Krueger et al. (2017) and many others before them have pointed out.

\section{A More Detailed Explanation of Why CRA Solves the Problems of Previous Two-Step Approaches}

We will now provide a more detailed explanation to illustrate that CRA is, in fact, an appropriate tool for investigating SE effects. The key problem of two-step approaches for investigating SE effects is that they lose information about subjects' self-views and criterion levels when discrepancy scores are computed in the first step of the analysis (Griffin et al., 1999; Ullrich, 2009; Zuckerman $\&$ Knee, 1996). For example, two people with very different levels of self-view and ability (e.g., Ray with $S=10, T=5$, and Sam with $S=6, T=1$ ) can be assigned the same algebraic 
Table 1: Example Data Set Reflecting a Self-View Effect but no SE Effect.

\begin{tabular}{lrrrr} 
& $\begin{array}{c}\text { Self-view } \\
\text { S }\end{array}$ & $\begin{array}{c}\text { Criterion } \\
\boldsymbol{T}\end{array}$ & $\begin{array}{c}\text { Discrepancy } \\
\text { score } \boldsymbol{S}-\boldsymbol{T}\end{array}$ & $\begin{array}{c}\text { Happiness } \\
\boldsymbol{H}\end{array}$ \\
\hline Ray & 10 & 5 & 5 & 20 \\
Sam & 6 & 1 & 5 & 12 \\
Tom & 6 & 5 & 1 & 12 \\
Kate & 5 & 1 & 4 & 10
\end{tabular}

Note. Happiness $H$ was constructed as $H=2 S+0 T$.

difference score $(S-T=5$; analogous observations can be made for any other SE operationalization, see Humberg et al., 2018). As a consequence, in the second step of the analysis, one cannot tell whether a positive correlation of $(S-T)$ and $H$ reflects an effect of the discrepancy $(S-T)$ or rather an effect of only one of its components $S$ and $T$. This approach can, for example, falsely indicate that $H$ is related to SE when there is in fact only a positive main effect of the self-view because the information that would be needed to identify the self-view effect is not available in the second step of the analysis.

Consider, for example, the simulated data in Table 1, which was constructed such that $H=2 S+0 T$. That is, $H$ is positively related to $S$ when $T$ is controlled for, but $H$ is unrelated to $T$ when $S$ is controlled for. To ease interpretation, we added no measurement error so that the data perfectly reflect the underlying effect. In this example, the two-step approach leads to a correlation of $\operatorname{Cor}(S-T, H)=.38$, and one would conclude that there is a positive SE effect. However, the data in fact contradict such an effect: Although Kate $(S-T=4)$ has a higher SE value than Tom $(S-T=1)$, Tom has a higher value on the outcome variable $\left(H_{\text {Tom }}=12, H_{\text {Kate }}=10\right)$. That is, the correlation of $(S-T)$ and $H$ falsely indicates a positive SE effect.

CRA solves this problem of the two-step approach. The reason is that CRA (a) preserves all information necessary to identify effects of the self-view and the criterion by including $S$ and $T$ as separate predictors in the regression model $\left(H=\beta_{0}+\beta_{1} S+\beta_{2} T\right)$ and (b) also makes use of all of this information by stating that the common constellation of two parameters must be inspected (i.e., two regression coefficients or two auxiliary parameters) before deciding whether the data indicate a positive SE effect. These properties, which contrast CRA against all previous approaches, enable researchers to unequivocally identify SE effects without misinterpreting simple self-view or criterion main effects as effects of SE (see OSF Material B at osf.io/e8p5r for the mathematical proof).

When applying CRA in practice, the differentiation between self-view main effects and SE effects can be explicitly observed (see also p. 10 in Humberg et al., 2018): A positive self-view effect (controlled for potential effects of $T$ ) is present when the coefficient $\beta_{1}$ of the selfview is positive $\left(\beta_{1}>0\right)$. This is the case for the example data in Table 1, where $\beta_{1}=2$ and $\beta_{2}=0$. For a positive SE effect, the two conditions " $a b s>0$ and $\beta_{1}-\beta_{2}>0$ " (Equation 3) must hold, where abs $>0$ must be significant and $\beta_{1}-\beta_{2}>0$ must hold numerically. For the example data, $a b s=0$ is not significantly positive, revealing that the data are not in line with a positive SE effect. That is, CRA, in contrast to the two-step approach, correctly indicates that only the self-view but not SE is positively related to happiness (see Humberg et al., 2018, for a more detailed explanation of CRA; see Tables 5, 6, and 7 in Humberg et al., 2018, for more examples).

\section{Conclusion}

In contrast to Krueger et al.'s (2017) conclusion, CRA provides a valid test for identifying effects of SE. Krueger et al.'s (2017) flawed assertion can be traced back to a misrepresentation of CRA as their argument focused on testing a single condition on the parameters of the regression model. CRA, by contrast, states that two conditions must be satisfied to indicate such an effect. These two conditions ensure that SE effects are clearly differentiated from self-view main effects. Thus, CRA overcomes critical limitations of prior intuitive but systematically biased two-step approaches, which have been typically applied to analyze the consequences of selfenhancement.

\section{Data Accessibility Statement}

No data was analyzed for this article.

\section{Notes}

${ }^{1}$ For some discrepancy measures (e.g., algebraic difference scores), the two-step approach has the additional problem of mistaking simple main effects of the reality criterion for SE effects. For simplicity and to be consistent with Krueger et al. (2017), we will not delve into this problem. CRA solves both problems because it differentiates SE effects from the main effects of the self-view and also from the main effects of the criterion.

${ }^{2}$ The source "Humberg et al. (2017)," which Krueger et al. (2017) cite for CRA, is the advance online version of the source "Humberg et al. (2018)," which we refer to in the present article.

${ }^{3}$ Here, we focus on the hypothesis of a positive SE effect because Krueger et al. (2017) also considered this hypothesis in their article. Naturally, CRA can also be used to test for a negative effect of SE, and the respective procedure is outlined in Figure 1 and in Humberg et al. (2018).

${ }^{4}$ CRA can be adapted to fit other operationalizations of SE (see below).

${ }^{5}$ Estimation of the regression model that forms the basis of CRA requires the classical assumptions of multiple regression. Moreover, as is typical for multiple linear regression models, estimation problems can arise when the predictor variables are highly collinear. In most domains in which SE effects are investigated, self-views and objective criteria are moderately correlated (e.g., around .3 for intelligence, see Mabe \& West, 1982; Zell \& Krizan, 2014; between .20 and .35 for personality, see Back \& Vazire, 2012; up to .13 for physical attractiveness, see Gabriel, Critelli, \& Ee, 
1994), such that multicollinearity should not occur in typical applications. We nonetheless recommend a pre-analysis to test for potential multicollinearity problems (e.g., by applying the variance inflation factor; VIF; Fox, 2016).

${ }^{6}$ CRA focuses on the associations of $S$ and $T$ with $H$, respectively, when the respective other predictor is controlled for (i.e., semi-partial correlation). By contrast, the raw correlations $\operatorname{Cor}(S, H)$ and $\operatorname{Cor}(T, H)$ are not relevant to the existence of an SE effect and are thus not considered in the CRA approach. Given that semipartial correlations can differ from raw correlations (e.g., see Horst, 1941; Smith, Ager, \& Williams, 1992), this implies that there can be an SE effect even if, for example, the raw correlation between $T$ and $H$ is positive (see also Humberg et al., 2018).

7 See also Footnote 11 in Humberg et al. (2018) for a detailed explanation of why testing $\beta_{1}-\beta_{2}>0$ or, equivalently, testing $\beta_{1}>\beta_{2}$, is not a valid test of an SE effect.

${ }^{8}$ To see that the set of CRA conditions " $\beta_{1}>0$ and $\beta_{2}<0$ " is not equivalent to the single condition " $\beta_{1}-\beta_{2}>0$," consider the example values $\beta_{1}=5$ and $\beta_{2}=3$, which satisfy $\beta_{1}-\beta_{2}>$ 0 but not the CRA conditions (because $\beta_{2}>0$ ).

${ }^{9}$ Krueger et al. (2017) referred to another observation during their critique of CRA, namely, that the regression weights $\beta_{1}$ and $\beta_{2}$ can correlate across studies. It is unclear why they assumed that this observation would have any implication on the validity of CRA. A nonzero correlation between coefficient estimates is a statistical consequence when the predictors are correlated. Whereas one should rule out the possibility that collinearity could be too high to reliably estimate the regression coefficients in the multiple regression model (see Footnote 5), correlated regression weights do not per se affect the validity of CRA.

10 The phrasing "negative $\beta_{2}$ " in this quote might indicate that the authors are suggesting that $\beta_{2}<0$ should be tested before the difference $\beta_{1}-\beta_{2}$ is considered (although this seems unlikely, given that the condition on $\beta_{2}$ does not show up in combination with $\beta_{1}-\beta_{2}$ in the rest of their critique). However, even if this were the case, the statement provides a misrepresentation of CRA because the conditions " $\beta_{2}<0$ and $\beta_{1}-\beta_{2}>0$ " are not equivalent to the conditions stated for the CRA approach: " $\beta_{1}>0$ and $\beta_{2}<0$." For example, the coefficients $\beta_{1}=-2, \beta_{2}=-3$ satisfy the first set of conditions but not the CRA conditions.

11 The question of whether it was theoretically justified to expect such effects is yet another area of discussion (see Humberg et al., in press).

\section{Funding Information}

This article was supported by the Open Access Publication Fund of the University of Münster.

\section{Competing Interests}

The authors have no competing interests to declare.

\section{Author Contributions}

Drafting the article or revising it critically for important intellectual content: SH, MD, FDS, KG, RH, MHWvZ, JJAD, SN, MDB

\section{References}

Asendorpf, J. B., \& Ostendorf, F. (1998). Is selfenhancement healthy? Conceptual, psychometric, and empirical analysis. Journal of Personality and Social Psychology, 74(4), 955-966. DOI: https://doi. org/10.1037/0022-3514.74.4.955

Back, M. D., \& Vazire, S. (2012). Knowing Our Personality. In: Vazire, S., \& Wilson, T. D. (Eds.), Handbook of SelfKnowledge, 131-156. Guilford Publications.

Bonanno, G. A., Field, N. P., Kovacevic, A., \& Kaltman, S. (2002). Self-enhancement as a buffer against extreme adversity: Civil war in Bosnia and traumatic loss in the United States. Personality and Social Psychology Bulletin, 28(2), 184-196. DOI: https://doi. org/10.1177/0146167202282005

Campbell, D. T., \& Kenny, D. A. (1999). A primer on regression artifacts. New York, NY, US: Guilford Press.

Church, A. T., Katigbak, M. S., del Prado, A. M., Valdez-Medina, J. L., Miramontes, L. G., \& Ortiz, F. A. (2006). A cross-cultural study of trait selfenhancement, explanatory variables, and adjustment. Journal of Research in Personality, 40(6), 1169-1201. DOI: https://doi.org/10.1016/j.jrp.2006.01.004

Colvin, C. R., Block, J., \& Funder, D. C. (1995). Overly positive self-evaluations and personality: Negative implications for mental health. Journal of Personality and Social Psychology, 68(6), 1152-1162. DOI: https:// doi.org/10.1037/0022-3514.68.6.1152

Dufner, M., Gebauer, J. E., Sedikides, C., \& Denissen, J. J. A. (2018). Self-enhancement and psychological adjustment: A meta-analytic review. Personality and Social Psychology Review. Advance online publication. DOI: https://doi.org/10.1177/1088868318756467

Edwards, J. R., \& Parry, M. E. (1993). On the use of polynomial regression equations as an alternative to difference scores in organizational research. Academy of Management Journal, 36(6), 1577-1613. DOI: https://doi.org/10.2307/256822

Fox, J. (2016). Applied regression analysis and generalized linear models. Thousand Oaks, USA: SAGE Publications.

Gabriel, M. T., Critelli, J. W., \& Ee, J. S. (1994). Narcissistic illusions in self-evaluations of intelligence and attractiveness. Journal of Personality, 62(1), 143155. DOI: https://doi.org/10.1111/j.1467-6494.1994. tb00798.x

Gramzow, R. H., Willard, G., \& Mendes, W. B. (2008). Big tales and cool heads: Academic exaggeration is related to cardiac vagal reactivity. Emotion, 8(1), 138144. DOI: https://doi.org/10.1037/1528-3542.8.1.138

Griffin, D., Murray, S., \& Gonzalez, R. (1999). Difference score correlations in relationship research: A conceptual primer. Personal Relationships, 6, 505518. DOI: https://doi.org/10.1111/j.1475-6811.1999. tb00206.x

Horst, P. (1941). The prediction of personal adjustment. Social Science Research Council Bulletin, 48, 1-156.

Humberg, S., Dufner, M., Schönbrodt, F. D., Geukes, K., Hutteman, R., Küfner, A. C. P., van Zalk, M. H. W., Denissen, J. J. A., Nestler, S., \& Back, M. D. (in press). Is accurate, positive, or inflated self-perception most advantageous for psychological adjustment? 
A competitive test of key hypotheses. Journal of Personality and Social Psychology. DOI: https://doi. org/10.31234/osf.io/9w3bh

Humberg, S., Dufner, M., Schönbrodt, F. D., Geukes, K., Hutteman, R., van Zalk, M. H. W., Denissen, J. J. A., Nestler, S., \& Back, M. D. (2018). Enhanced versus simply positive: A new condition-based regression analysis to disentangle effects of self-enhancement from effects of positivity of self-view. Journal of Personality and Social Psychology, 114(2), 303-322. DOI: https://doi.org/10.1037/pspp0000134

John, O. P., \& Robins, R. W. (1994). Accuracy and bias in self-perception: Individual differences in selfenhancement and the role of narcissism. Journal of Personality and Social Psychology, 66(1), 206-219. DOI: https://doi.org/10.1037/0022-3514.66.1.206

Krueger, J. I., Heck, P. R., \& Asendorpf, J. B. (2017). Selfenhancement: Conceptualization and Assessment. Collabra: Psychology, 3(1), 28. DOI: https://doi. org/10.1525/collabra.91

Krueger, J. I., \& Wright, J. C. (2011). Measurement of selfenhancement (and self-protection). In: Alicke, M. D., \& Sedikides, C. (Eds.), Handbook of self-enhancement and self-protection, 472-494. Guilford Press.

Kurt, A., \& Paulhus, D. L. (2008). Moderators of the adaptiveness of self-enhancement: Operationalization, motivational domain, adjustment facet, and evaluator. Journal of Research in Personality, 42(4), 839-853. DOI: https://doi.org/10.1016/j.jrp.2007.11.005

Kwan, V. S. Y., John, O. P., Kenny, D. A., Bond, M. H., \& Robins, R. W. (2004). Reconceptualizing individual differences in self-enhancement bias: An interpersonal approach. Psychological Review, 111(1), 94-110. DOI: https://doi.org/10.1037/0033-295X.111.1.94

Kwan, V. S. Y., John, O. P., Robins, R. W., \& Kuang, L. L. (2008). Conceptualizing and assessing selfenhancement bias: A componential approach. Journal of Personality and Social Psychology, 94(6), 1062-1077. DOI: https://doi.org/10.1037/0022-3514.94.6.1062

Mabe, P. A., \& West, S. G. (1982). Validity of selfevaluation of ability: A review and meta-analysis. Journal of Applied Psychology, 67(3), 280-296. DOI: https://doi.org/10.1037/0021-9010.67.3.280

Moore, D. A., \& Healy, P. J. (2008). The trouble with overconfidence. Psychological Review, 115(2), 502-517. DOI: https://doi.org/10.1037/0033-295X.115.2.502

Paulhus, D. L. (1998). Interpersonal and intrapsychic adaptiveness of trait self-enhancement: A mixed blessing? Journal of Personality and Social Psychology, 74(5), 1197-1208. DOI: https://doi. org/10.1037/0022-3514.74.5.1197

Paulhus, D. L. (2008). Self-presentation of personality: An agency-communion framework. In: John, O. P., Robins, R. W., \& Pervin, L. A. (Eds.), Handbook of personality: Theory and research, 3rd ed., 492-517. New York, NY: Guilford Press.

Robins, R. W., \& Beer, J. S. (2001). Positive illusions about theself:Short-term benefits and long-term costs. Journal of Personality and Social Psychology, 80(2), 340-352. DOI: https://doi.org/10.1037/0022-3514.80.2.340

Robins, R. W., \& Paulhus, D. L. (2001). The character of self-enhancers: Implications for organizations. In: Roberts, B. W., \& Hogan, R. (Eds.), Personality psychology in the workplace, 193-219. American Psychological Association. DOI: https://doi.org/10.1037/10434-008

Sedikides, C., \& Gregg, A. P. (2008). Self-enhancement: Food for thought. Perspectives on Psychological Science, 3(2), 102-116. DOI: https://doi. org/10.1111/j.1745-6916.2008.00068.x

Smith, R. L., Ager, J. W. J., \& Williams, D. L. (1992). Suppressorvariables in multiple regression/correlation. Educational and Psychological Measurement, 52, 17-29. DOI: https://doi.org/10.1177/001316449205200102

Taylor, S. E., \& Brown, J. D. (1988). Illusion and wellbeing: A social psychological perspective on mental health. Psychological Bulletin, 103(2), 193-210. DOI: https://doi.org/10.1037/0033-2909.103.2.193

Taylor, S. E., Lerner, J. S., Sherman, D. K., Sage, R. M., \& McDowell, N. K. (2003). Are self-enhancing cognitions associated with healthy or unhealthy biological profiles? Journal of Personality and Social Psychology, 85(4), 605-615. DOI: https://doi. org/10.1037/0022-3514.85.4.605

Ullrich, J. (2009). Reconsidering the "relative" in relative ingroup prototypicality. European Journal of Social Psychology, 39, 299-310. DOI: https://doi. org/10.1002/ejsp.540

Zell, E., \& Krizan, Z. (2014). Do people have insight into their abilities? A metasynthesis. Perspectives on Psychological Science, 9(2), 111-125. DOI: https://doi. org/10.1177/1745691613518075

Zuckerman, M., \& Knee, C. R.(1996). The relation between overly positive self-evaluation and adjustment: A comment on Colvin, Block, and Funder (1995). Journal of Personality and Social Psychology, 70(6), 1250-1251. DOI: https://doi.org/10.1037/0022-3514.70.6.1250 
Peer review comments

The author(s) of this paper chose the Open Review option, and the peer review comments are available at: http://doi.org/ 10.1525/collabra.137.pr

How to cite this article: Humberg, S., Dufner, M., Schönbrodt, F. D., Geukes, K., Hutteman, R., van Zalk, M. H. W., Denissen, J. J. A., Nestler, S., \& Back, M. D. (2018). Why Condition-Based Regression Analysis (CRA) is Indeed a Valid Test of Self-Enhancement Effects: A Response to Krueger et al. (2017). Collabra: Psychology, 4(1): 26. DOl: https://doi.org/10.1525/collabra.137

Senior Editor: Don Moore

Submitted: 07 February 2018 Accepted: 07 July 2018 Published: 23 July 2018

Copyright: ( $) 2018$ The Author(s). This is an open-access article distributed under the terms of the Creative Commons Attribution 4.0 International License (CC-BY 4.0), which permits unrestricted use, distribution, and reproduction in any medium, provided the original author and source are credited. See http://creativecommons.org/licenses/by/4.0/. 